\title{
Post- Partum Variables Among Breastfeeding Women and Its Relative Effect on Maternal and Infant Health in Ekiti State Nigeria
}

\author{
Ogunlade Joseph Olurotimi (Ph,D) Babatunde Christianah Adekemi (Mrs) \\ Dept.of Educational Psychology\&Counselling, College of Education, Ikere Ekiti
}

\begin{abstract}
The study examined Post-Partum Variables, Maternal and Infant Health among breastfeeding Women in Ekiti State Nigeria. The descriptive research design of the survey type was used for this study. The population for the study was made up of women with breastfeeding experience in Ekiti State Nigeria. The sample consists of 278 women sampled from three local government area in Ekiti State Nigeria. Multistage sampling technique was used to select the sample. The research instrument used to collect data from the respondents was a self-designed questionnaire titled Post-Partum Variables, Maternal and Infant Health Questionnaire (PVMIHQ). The instrument was valid and reliable; it had a construct validity correlation coefficient of 0.74 and a reliability coefficient of 0.86 which were obtained at 0.05 level of significance. The study revealed that women are well informed about PostPartum Variables on Maternal and Child Health due to series of information acquired through lectures, seminars and workshops during the ante natal period in their various maternity centres and hospitals which indicated that they were duly prepared for the pains and the gains after birth.
\end{abstract}

Keywords: Post Partum, Variables, Breastfeeding, Marternal, Infant.

DOI: $10.7176 / \mathrm{JHMN} / 73-05$

Publication date: April $30^{\text {th }} 2020$

\section{Introduction}

Breastfeeding is the act of feeding the baby from the breast. According to WHO (2012) Breastfeeding confers substantial health benefits on both mother and child. For the infant, the principal advantages of breast milk are nutritional and immunological. For the mother, breastfeeding encourages the involution of the uterus and thus the rapid return of uterine tomb which helps the mother to regain her natural shape.

To achieve this, during pregnancy, the breasts go through distinct changes as they prepare for nourishing a new infant. Within two or three days after a woman might have given birth to a new baby her breasts *begin to produce milk. This process of milk production is known as lactation. This period as well coincides with postpartum period which begins with the first 1-2 hours after birth when mother and infant are recovering from birth and become (re) acquainted with each other this moment is mostly associated with pain fundus, bladder, lochia, perineum/dressing. UNICEF/WHO (2010) added that it is also the time to make the most of the first infant feeding opportunity which is characterized with observe physical response of mom and baby, nutritional status of mom and baby, post anesthesia/analgesia status of mom and baby when feelings should return to legs which continues for about 6 weeks, or so

Breastfeeding behaviour according to Foster (2006), is a social phenomenon related to breastfeeding activities, which are under the control of norms and standards of social practices and expectations. They constitute activities which provide support for the mothers' choice to breastfeeding based on the behaviour that is healthiest for her and her new born, giving rise to what is commonly referred to as exclusive breastfeeding and supplementary breastfeeding practice. It is observed that the incidence and duration of breastfeeding and its associated practice of postpartum amenorrhea seems to be declining among women in south west Nigeria, which tends to shorten the length of birth interval.

There is several knowledge - related factors which seems to affect breastfeeding. Such as practice of postpartum amenorrhea, Postpartum depression and the strength of the mother's desire for a healthy infant combined with her knowledge about the benefits of breastfeeding to the child and her strong desire for good health and fertility (Ogunlade, 2010). Hence this research work examined postpartum depression, breastfeeding initiation and duration.

According to MedlinePlus (2017) Postpartum depression (PPD) is a complex mix of physical, emotional, and behavioral changes that happen in a woman after giving birth. This is tailored to the DSM-5, a manual used to diagnose mental disorders which explain further that PPD is a form of major depression that has its onset within four weeks after delivery. The diagnosis of postpartum depression is based not only on the length of time between delivery and onset, but also on the severity of the depression. The term describes a range of physical and emotional changes that many new mothers experience. It is on record that this nagging experience has been adjudged by different researchers as one of the reasons for decrease in breastfeeding duration and a ruse to exclusive breastfeeding but the good news is that postpartum depression can be treated with medication and counselling (NIMH, 2016). 
The experiences of postpartum depression are similar to what happens normally following childbirth. They include difficulty in sleeping, appetite changes, excessive fatigue, decreased libido, and frequent mood changes. However, these are also accompanied by other symptoms of major depression, which are not normal after childbirth, and may include depressed mood; loss of pleasure; feelings of worthlessness, hopelessness, and helplessness; thoughts of death or suicide or thoughts or hurting someone else.

Hilton (2016) opined that a number of factors can increase the risk of postpartum depression, including: a history of depression prior to becoming pregnant or during pregnancy, the mother's age at the time of pregnancy, the number of children the woman has, a history of depression or premenstrual dysphoric disorder (PMDD); having limited social support; living alone; and, marital conflict. Hilton suggests that the younger a woman is, the higher the risk of ambivalence about the pregnancy, and the more children a woman has, the more likely she is to be depressed in a subsequent pregnancy. Fredrick (2016), suggested some tips that can help a new mother to cope with bringing home a newborn, such as asking for help, letting others know how they can help her, being realistic about her expectations for herself and baby, exercising within the limits of doctor's restrictions, and getting out of the house for a break. Mothers should expect some good days and some bad days, follow a sensible diet, avoid alcohol and caffeine, foster the relationship with their partner, make time for each other, keep in touch with family and friends, do not isolate themselves, limit visitors when first going home, screen phone calls, and sleep or rest when baby sleeps.

Postpartum depression is treated differently depending on the type and severity of a woman's symptoms. Treatment options include anti-anxiety or antidepressant medications, psychotherapy, and participation in a support group for emotional support and education. In the case of postpartum psychosis, drugs used to treat psychosis are usually added. Hospital admission is also often necessary. If you are breastfeeding, don't assume that you can't take medication for depression, anxiety, or even psychosis. You are advice to talk to your doctor. Under a doctor's supervision, many women take medication while breastfeeding. This is a decision to be made between you and your doctor. It is noteworthy that untreated postpartum depression can be dangerous for new moms and their children. A new mom should seek professional help when, symptoms persist beyond two weeks, she is unable to function normally and can't cope with everyday situations, she has thoughts of harming herself or her baby where she is feeling extremely anxious, scared, and panicked most of the day.

Meanwhile, postpartum experience should not preclude breastfeeding decision because it is on record that shortly before delivery the levels of estrogens and progesterone fall in order to stimulate the pituitary gland produce lactogenic hormone which act on the acini's cell in the breast to start the production of milk (WHO,2010) UNICEF (2012) explain that in the beginning of breast feeding or the point of initiation the experience may be strange, tingling and exciting but in a matter of time mother and baby will surely find a comfortable and enjoyable routine naturally beneficial to both.

The report of Ogunlade (2010) alleviated the complaints of breastfeeding women about insufficient breast milk due to issues like breast size and shapes as just a common bodily worries that has no direct effect in the biological functions of the system, he explained that at puberty the female breast under the influence of homones called oestrogen starts showing famine contour with the development of secondary tissue and are richly supply with blood. The breast develops in different sizes and shapes just as individuals different from person to person. The following are the contradictions to woman health in a few cases have been agreed upon that exceptions be granted to breastfeeding: cases of HIV infections in mother, some medical reason where the mother is on a particular drug, cases of tuberculosis, psychiatric disorders, cases of diabetes mellitus were stillbirth or neonatal death occurs anathesis (UNICEF, WHO 2010)

The inability of women to change their behaviour towards the improvement of breastfeeding practices to ensure a sound health for the infant and the mother is the major concern of the researcher. Observation also reveals that the idea of women breastfeeding a child for 12 months is now seen as antisocial and primitive. If nursing mothers continue to reduce the incidence and quality of breastfeeding practice it may be dangerous and disastrous to the health of the mother and the child, also it may as well lead to short birth interval and have an adverse effect on the population.

\section{Review of Related Literature}

Essentials of Breastfeeding and Fertility

Webledon (2018). in Traci (2018). reported that breastfeeding provides a natural delay in women's return to fertility. He explained that baby's sucking controls the mother's ovulation, the more the baby has a need to suck, the less ready he is to be displaced by another. The less baby has a need to suck, the more ready and able he is to cope with a new brother or sister. Woman Art of Breastfeeding noted that almost all the mothers who are fully breastfeeding their babies are free from menstrual periods for at least the first six months. This process is called Lactational Amenorrhea which is guaranteed by full breastfeeding that means the baby relies completely on the mother for nourishment and for all of his sucking needs. It was concluded here that baby's frequent sucking or nursing inhibits the release of hormones that causes mothers body to begging the monthly preparation for a new pregnancy. At this period ovulation does not take place and you do not have menstrual periods. 
According to Kippely (1999), the biggest effect of breastfeeding on menstrual cycles in guaranteed through ecological breastfeeding. Kippley (1999), explained seven tenets of ecological breastfeeding to mean: -

(1) Exclusive breastfeeding (no other liquid or solid food from any other source enters the infant's mouth) for the first six months of life.

(2) Comfort your baby at the breast.

(3) Don't use bottles and pacifiers

(4) Share sleep with your baby for night feedings

(5) Share sleep with your baby for daily nap feelings

(6) Nurse frequently day and night and avoid schedules

(7) Avoid any practice that restricts nursing or separate you from your baby.

Lawrence and Lawrence (1994) reported that researcher in Georgetown University developed the Lactational Amenorrhea Method (LAM) of birth control to qualify exactly under birth circumstance breastfeeding is sufficient birth control.

Three conditions were opined such as:

(1) Baby is less than six months old

(2) The mother's period has not yet returned

(3) Baby is exclusively breastfed. (with no pacifiers, supplemental bottle, or solid food) and nurses on demand day and night. She concluded that it is important that mothers don't expect casual breastfeeding to limit their fertility.

WHA (1992) in her own study asserted that breastfeeding is a good choice for the mother who prefers a natural family planning does not want to take hormonal medication in the early months postpartum but 3 criteria must be that:

(1) The baby should not be receiving any supplemental foods or artificial formula,

(2) The baby must be less than 6 months old and

(3) The mother must not have resumed her menstrual cycle.

It was equally explained that ovulation in the non-lactating woman may occur as early as three weeks postpartum and that the risk of ovulation in the high frequency of breastfeeding, amenorrhea in women is then one to two percent because frequent nursing stimulates proclatin levels, which in turn suppress the surge of the follicle stimulating and luteanizing hormones so that effective ovulation does not occur.

The Lactational Amenorrhea method (LAM): Another Choice for Mothers

Labbok (1993) reported that at the six-week postpartum check up, the new mother is often told that she must begin contraceptive use immediately to avoid a pregnancy too soon. But what method should she choose? Research substantiates that women worldwide believe breastfeeding is associated with fertility reduction, but the question remains: How to use it effectively? The Lactational Amenorrhea Method (LAM) answers that question.

The Lactational Amenorrhea Method (LAM) is defined as a newly developed interim family planning method that is based on utilization of Lactational infertility for protection from pregnancy. The Lactational Amenorrhea Method (LAM) provide optimal infant nutrition, enhances immunity, prevents formula-related illness, and physiologically promotes mother-child interaction while simultaneously providing safe and effective temporary child spacing. It may be used for up to six months postpartum during full or nearly full breastfeeding and amenorrhea and has been shown in clinical trial to be 99 percent effective (Gray, WHO, (2002).

The Lactational Amenorrhea Method (LAM) was developed as a result of a meeting held at Georgetown University. (Shelton, 1992). it is designed to bring the health and fertility benefits of breastfeeding to the attention of family-planning providers and demographers. A mother is asked these three questions: Is your infant less than six months old? Are you amenorrheic? Are you fully or nearly fully breastfeeding? If she can answer yes to all the three questions, she is counselled that her risk of pregnancy is less than two percent and she does not need a complementary family-planning method yet. She is also told that if any of these three parameters changes, she should introduce a complementary form of family planning to achieve this same low risk of pregnancy (Kenedy (2006).

Lactational Amenorrhea Method (LAM) proved highly efficacious, with a pregnancy rate of less than $1 / 2$ of 1 percent by six-month life table. The intervention more than doubled the percent of women who achieved six months of meeting Lactational Amenorrhea Method (LAM) criteria. At six months postpartum, family planning coverage had increased from 78 percent to 91 percent with the inclusion of Lactational Amenorrhea Method (LAM) in the cafeteria of methods available. It was also argued by Howie (2006) that pregnancy rates remained lower for the intervention group for over a year, and the percent who were breastfeeding remained higher long after the intervention was over. The duration of amenorrhea was also extended. Where the control group performed similarly to other published studies on the duration of amenorrhea during full breastfeeding among similar women. The intervention group according to Hoser (2008) had longer duration of amenorrhea, even when comparing the full breastfeeders in each group. Clearly, optimizing breastfeeding practices, even among full breastfeeders, 
extends the duration of amenorrhea and the impact of breastfeeding on fertility, even after Lactational Amenorrhea Method (LAM) use has ceased.

Tilapia (2005) noted that much work remains to fully educate providers of health care on the use of the method, especially those who doubt women's ability to monitor their own behaviour. Lactational Amenorrhea Method (LAM) may be offered to women who prefer to postpone introducing a complementary family planning method postpartum.

In spite of the versatility of Lactational Amenorrhea Method (LAM), research indicates that the Lactational Amenorrhea Method (LAM) guidelines are very conservative and that each of the three parameters has considerable flexibility. Horton 2004 affirmed that six months is an arbitrary time period; we know that continuing to breastfeed prior to each supplemental feed can extend amenorrhea and infertility. Full breastfeeding is not mandatory. Although a recently published manuscript seems to say that any breastfeeding will do during the first six months, it is clear that more intensive breastfeeding is associated with longer durations of infertility. Even using menses as an indication of fertility return has some flexibility; the first ovulation is often associated with inadequacies in the luteal phase and other hormonal parameters. Based on worldwide experiences with relying on Lactational Amenorrhea beyond six months, we are now exploring what we call Lactational Amenorrhea Method (LAM) II, a new more flexible method (Morgan (2007).

In conclusion the unique side effects of Lactational Amenorrhea Method (LAM), improved infant and maternal health and satisfied family planning workers, contribute in yet another way to the health of the community. A mother's postpartum family planning choices now include a reliable interim method based on the behaviour that is healthiest for her and her new born.

Factors Influencing Breastfeeding Duration and Birth Spacing

As we have noted above, considerable research has been conducted in developing countries 2000 on the potential benefits of breastfeeding, particularly its positive effects on infant health (Popkin, (1983) McCan, (1981); and Winikoff, (2000); and on the emotional development and demographic consequences of breastfeeding (Simpson - Herbert and Huffman, (1976);

A significant body of research has been developed on the delineation and analysis of factors influencing breastfeeding and child spacing. Most analyses have attributed recent breastfeeding declines in developing countries to infant food industry marketing pressures and to biases towards bottle feeding from the medical professionals. Some literatures have attributed the decline to the erosion of traditional value and modes of living (such as declines in the extended family system thought to have supported breastfeeding or to increase urbanization and the associated tensions that inhibit the let-down reflex which makes milk available to the sucking infant and are related to the insufficient mil syndrome (Popkin, (1983). Probably these factors have played a role, but changes in infant feeding patterns in developing countries are occurring simultaneous with considerable social and economic changes. Most previous empirical research studies have been univariate, thereby ignoring most socioeconomic forces such as modernization and changes in women's roles that affect breastfeeding.

Numerous factors appear to affect breastfeeding decisions, although their relative importance varies both across and within countries. Since the mother is the ultimate actor and usually the key decision maker with regard to breastfeeding, behavioural and biological factors related to her are of central concern. Factors external to the mother and the household such as cultural norms, media exposure and health professionals exert powerful direct and indirect pressure on breastfeeding behaviour. (Kramer \& Kakuma, (2009),

The decision to breastfeed is affected by the compatibility of the mother's job with child care and breastfeeding. The hours when employment is available, its closeness to the mother's home, and the availability of work breaks that could be used to return home to breastfeed on the one hand or her ability to take the infant with her while she works and periodically nurse it clearly influence breastfeeding practices. While many researchers refer to the relationship between maternal work and breastfeeding decisions (UNICEF, (2016) and WHO (2016), few empirical studies on this topic have been conducted, and this has been mostly in urban areas, in one study, Popkin and Solon (1983) found a significant association between the location of the mother's job and her breastfeeding behaviour. They found in particular that infants of working women were more likely to be mixed fed. (Butz and Da Vanzo (1981) found that the mother is more likely to breastfeed and for a longer time in Malaysia if her job were in agriculture. However, another analysis in Malaysia found that women who were involved in sales $r$ productive activities including dressmakers, food and beverage makers or weaves were more likely to have children less than ten years of age with them when they worked than those involved in other types of economic activities including professional occupations, management, clerical work and service occupations (Power and Luck (2009). They did not however link these child care compatibility variables with their breastfeeding research.

\section{Statement of the Problem}

It is on record that the nagging experience of postpartum depression has been adjudged by different researchers as one of the reasons for decrease in breastfeeding duration and a ruse to exclusive breastfeeding (NIMH, 2016). The 
researcher also observed that women in Ekiti State Nigeria seems breastfeeding far below the submission of Bellagio Consensus 1995, WHO (2004), UNICEF (1992) that women must breastfeed their babies exclusively for the first six month of life. Meanwhile records has it that a reduction in the initiation and duration of breastfeeding may be dangerous to the health of the mother and the child .Hence, this research work specifically examined the physical, emotional, and behavioral changes that happen in a woman after giving birth and its associated effect on breastfeeding initiation and duration.

\section{Purpose of the Study}

The reason behind this study is to expose mothers to the realistic expectations of their infant's breastfeeding behavior to support the decision to breastfeed and avoid unnecessary supplementation. Specifically, the objective was to strengthen the mother's desire for a healthy infant combined with her knowledge about the benefits of breastfeeding to the child and her strong desire for good health by encouraging them to initiate breastfeeding shortly after birth and ensure exclusive breastfeeding for six months all physical and emotional health conditions notwithstanding.

\section{Hypotheses}

This study was guided by the following hypotheses and were tested at 0.05 level of significance.

$\mathrm{HO} 1$. There is no significant difference in the breastfeeding initiation and duration among Ekiti State women based on their physical changes after birth.

HO2. Emotional changes after birth among women in Ekiti State Nigeria will not significantly influence her breastfeeding initiation and duration.

\section{Methodology}

The research design used was the descriptive research design of the survey type. The population for the study was made up of women with breastfeeding experience in Ekiti State. The sample consisted of 278 women selected from three local government area of Ekiti state Nigeria. Multistage random sampling technique were used. The research instrument used to collect data from the respondents was a self-designed questionnaire titled Post-Partum Variables, Maternal and Infant Health Questionnaire (PVMIHQ) The research instrument consisted of two sections A and B. Section A consist of social characteristic of the respondent such as religion, sex of their children, age, location of residence, educational qualifications and some background information such as birth interval, method of feeding. Section B consists of 20 items on the physical, emotional balance and the readiness to initiate breastfeeding. The instrument was construct validated and a split half reliability coefficient of 0.87 was obtained. Research assistants and the researcher administered the questionnaire. The Data was analysed using inferential statistic such as ANOVA to test the hypotheses at 0.05 level of significance.

\section{Result Presentation}

\section{Hypothesis 1}

There is no significant difference in the breastfeeding initiation and duration of Ekiti State Nigerian women based on their physical changes after birth.

Table 1: Chi-square test for influence in the breastfeeding initiation and duration of Ekiti State women based on their physical changes after birth.

\begin{tabular}{|l|l|l|l|}
\hline Test & Value & Df & Asymp. Sig. (2-sided) \\
\hline Chi-Square & 19.797 & 16 & .002 \\
\hline Likelihood Radio & 19.332 & 16 & .000 \\
\hline Linear-by-Linear & .338 & 1 & .001 \\
\hline Association & & & \\
\hline No of Valid Cases & 278 & & \\
\hline
\end{tabular}

The result presented in table 7.1 shows that $\mathrm{x} 2$ value for the influence of breastfeeding initiation and duration based on physical changes after birth 19.797. The p- value $0.000<0.005$ indicates a statistically significant influence of breastfeeding initiation and duration based on physical changes after birth. Based on this, the null hypothesis was rejected. Therefore, it was concluded that physical changes after birth has a moderate influence on breastfeeding initiation and duration

\section{Hypothesis 2}

Emotional changes after birth among women in Ekiti State Nigeria will not significantly influence her breastfeeding initiation and duration. 
Table2: Chi-square test for influence of Emotional changes after birth among women on her breastfeeding initiation and duration.

\begin{tabular}{|l|l|l|l|}
\hline Test & Value & \multicolumn{1}{|c|}{ Df } & Asymp.Sig. (2-sided) \\
\hline Chi-Square & 12.501 & 16 & .009 \\
\hline Likelihood Radio & 12.665 & 16 & .006 \\
\hline Linear-by-Linear & .407 & 1 & .004 Association \\
\hline No of Valid Cases & 278 & & \\
\hline
\end{tabular}

The result presented in table 7.2 shows the $\mathrm{x} 2$ value for the influence of Emotional changes after birth among women as 12.501. The p-value $=.009>.05$ indicate a statistically significant influence on her breastfeeding initiation and duration. Based on this, the null hypothesis that Emotional changes after birth among women in Ekiti State Nigeria will not significantly influence her breastfeeding initiation and duration was rejected. Therefore, it was concluded that Emotional changes after birth among women significant influence her breastfeeding initiation and duration.

\section{Discussion of the Findings}

From the results, it is evident that physical changes affect breastfeeding initiation and duration which is in tandem with the submission of Fieve [3] that this moment is mostly associated with pain fundus, bladder, lochia, perineum/dressing. Very importantly, it is the time to make the most of the first infant feeding opportunity, which is premised on the observed physical response of mom and baby. The result from this work also indicated that emotional changes after birth among women have significant influence on her breastfeeding initiation and duration. This agrees with the submission of MedlinePlus (2017), that Postpartum depression (PPD) is a complex mix of physical, emotional, and behavioral changes that happen in a woman after giving birth. These changes are not normal after childbirth, and may include depressed mood; loss of pleasure; feelings of worthlessness, hopelessness, and helplessness; thoughts of death or suicide or thoughts or hurting someone else.

The good news according to NIMH, (2016) is that postpartum depression can be treated with medication and counselling. This could be substantiated with the submission of UNICEF (1992) who explained that in the beginning of breast feeding or at the point of initiation the experience may be stranged, tingling and exciting but in a matter of time mother and baby will surely find a comfortable and enjoyable routine naturally beneficial to both. The report of Ogunlade (2010) also alleviated the complaints of breastfeeding women about insufficient breast milk due to issues like breast size and shapes, as just a common bodily worries that have no direct effect in the biological functions of the system. He explained that at puberty the female breast under the influence of hormones called oestrogen starts showing famine contour with the development of secondary tissue and which are richly supply with blood. The breast develops in different sizes and shapes just as individuals are different from person to person.

Essential for discussion is that to guarantee or help mother to cope with bringing home a newborn, she needs to let others know how they can help her, be realistic about her expectations for herself and her baby, exercise within the limits of any restrictions her doctor may place on her level of activity. She could take a walk, and get out of the house for a break, expect some good days and some bad days, follow a sensible diet; avoid alcohol and caffeine, foster the relationship with her partner, make time for each other, keep in touch with family and friends, do not isolate herself, limit visitors when she first goes home, screen phone calls, and sleep or rest when her baby sleeps (NIMH 2010).

\section{Conclusions}

Therefore, it was concluded that physical changes after birth has a moderate influence on breastfeeding initiation and duration and that emotional changes after birth among women significantly influenced her breastfeeding initiation and duration.

\section{Recommendations}

Basing on the results it was recommended that mothers should commence breastfeeding immediately after delivery. In case of postpartum challenges she should adhere strictly to doctors' advice and counselling from professionals. It was also recommended that she should not nurture any fear and be optimistic about regaining her good health and shape no matter her condition.

\section{References}

American Psychiatric Association (2000). Diagnostic and Statistical Manual of Mental Disorders: DSM-IV-TR, American Psychiatric Pub.

Butz, F.F. \& Devanzo E.D., (1981) 'Determinants of breast-feeding and weaning patterns in Malaysia. Paper presented at the $50^{\text {th }}$ annual meeting of the Population Association of America, Washington DC; March 2628.

Fieve, R, (2006) MD. Bipolar II, Rodale Books.

Foster, M.S., (2016) Women's Decision about Breastfeeding and Maternal Employment. Journal of Marriage and 
the Family 58 239-251.

Gray, R.H. Campbell, R. \& Apelo, S. (2002) The Risk of Evaluation during Lactation. Lancet; 335, 25-29.

Horton S. (2004) Lactational Amenorrhea and the Recovery of Ovulation and Fertility in Fully Nursing Chilean Women Lancet.

Hoser, F.H. (2008) Contraceptive Efficacy of Lactational Amenorrhea. Lancet.

Howie, P (2006) Effect of Breastfeeding patterns on human birth intervals. Journal of Reproductive Fertility.

Kenedy, K \& Viness C. (2006) Contraceptive efficacy of Lactational Amenorrhea Lancet: 339, 227-229

Kippley, S.M (1999), Breastfeeding and Natural Child Spacing. Cincinnati, OH: CCL, 125-128

Kramer MS, Kakuma R. (2009) Optional Duration of Exclusive Breastfeeding, Cochrane Review, The Cochrane Library. 140-144.

Labbok, M. \& Krasovec, K. (2003) Towards Consistency in Breastfeeding Definitions. Stud fam plan 21(4) 226 30.

Labbok, (1993) M.H Breastfeeding and Conception. New Eng J Med 308:51

Lawrence, R \& Lawrence, R. Breastfeeding: A Guide for the Medical Profession, New Jersey: Elseriver).

McCan, M.F. (1981) "Breastfeeding Fertility and Family Planning”. Population Report Series J.: 24

MedlinePlus, (2017) "Medical Encyclopaedia: Post-partum Depression,".

Morgan J. (2007) Clinical Study of the Lactational Amenorrhea Method for Family Planning. Lancet.

National Institute of Mental Health, (2010) "What is Depression?".

National Institute of Mental Health, (2010) "Depression: What Every Woman Should Know,".

National Institutes of Health News in Health: (2000)"Understanding Post-Partum Depression, Common but Treatable,".

Ogunlade J. O (2010) "Breastfeeding and Child Spacing Among Women in Nigeria". Ph.D Thesis Ekiti State University. Ado- Ekiti, Nigeria,

Ojofeitimi, E.O. (1981) Breastfeeding Patterns in a Nigerian Maternity Centres Clinical Paediatrics 20, $412-414$

Popkin, B.M., (1983) "Breastfeeding Determinants in low-income Countries" Medical Anthropology. 7(1): 1-31.

Power M, Lock E, Chapin J, Klein L, Schulkin J (2009) The Effort to Increase Breastfeeding: Do Obstericians, in the Forefront, Need help? Journal of Reproductive Medicine 48, 72-78.

Shelton, K, (1992). Guideline for Breastfeeding in Family Planning and Child Survival Programs. Institute for Reproductive health, Georgetown University.

Simpson-Herbert, M., \& Chris. M. (1976) “Breast-feeding”, Studies in Family Planning, 12, 125-133.

Tilapia, F. (2005) The Impact of a hospital and clinic-based breastfeeding promotion program. Journal of Tropical Paediatric in Press.

UNICEF, (1992) Lactational Amenorrhea Method (LAM) Publication of Georgia University, Institute for Reproductive Health.

UNICEF, (2012) Lactational Amenorrhea Method (LAM) Publication of Georgia University, Institute for Reproductive Health.

UNICEF/ WHO, (2010) Factors influencing decision to breastfeeding of Advance Nursing. 369-468.

Webledon M.D (2018) Medical Reference Reviewed by Traci C. Johnson, MD on April 11, 2011

WHA, (1992) Report on the state of the World Health Feeding Policy. HIV and Infant Feeding,

WHO, (2002) The World Health Report Make every mother and child count. A special report of WHO Geneva. WHO Global Data Bankon Breastfeeding. Geneva, World Health Organization.

WHO, (2004) Essentials of Contraceptive Technology, John Hopkins Program for International Education in Reproductive Health.

WHO, (2012) Preliminary report of the WHO collaborative study on breastfeeding (MCH/79.3): Geneva, Winikoff, B. (2000) "Nutrition, and Health: Some Implications for Policy". Science, 4344, 895-902. 